\title{
Abundância e distribuição das larvas de peixes no Lago Cataláo e no encontro dos rios Solimóes e Negro, Amazonas, Brasil
}

\author{
Rosseval Galdino LEITE ${ }^{1}$, José Vagner Valente da SILVA ${ }^{1}$, Carlos Edwar FREITAS²
}

\section{RESUMO}

A abundância e a distribuição mensal de larvas de peixes no Lago Catalão, situado próximo ao encontro dos rios Solimões e Negro, foram avaliadas entre os meses de janeiro e maio de 2001. As amostras foram coletadas com uma rede de ictioplâncton cônicocilíndrica (350 $\mu \mathrm{m}$ de malha, Ø 0,3 m e $2 \mathrm{~m}$ de comprimento), com um fluxômetro acoplado na boca da rede para a obtenção do volume de água filtrado pela mesma. As variáveis categóricas consideradas foram: posição no lago (margem e centro), profundidade (superfície e fundo) e turno (diurno e noturno); tendo sido realizada a Análise Fatorial de Correspondência (AFC) usando a densidade larval (larvas. $\mathrm{m}^{-3}$ ) como variável resposta e os meses como descritores. A AFC discriminou a ocorrência de larvas por unidade experimental entre o mês de janeiro e os demais meses, com 58,15\% de inércia, e a densidade larval mudou conforme a posição no lago e o período do dia. A maior parte das larvas era protolarva e alimentava-se na margem, tanto na superfície quanto no fundo do lago. Concluiu-se que o Lago Catalão é um importante berçário para as larvas dos peixes que desovam no rio Solimões e também para as larvas dos peixes que desovam no próprio lago.

PALAVRAS-CHAVE

Rio Solimōes, Lago Catalão, larvas de peixes, abundância, distribuição, alimentação.

\section{Abundance and distribution of fish larvae in Cataläo Lake and in the confluence of the Solimoes and Negro River, Amazon, Brazil}

\section{ABSTRACT}

The abundance and monthly distribution of fish larvae in Cataläo Lake, situated near the confluence of the Solimōes and Negro River was

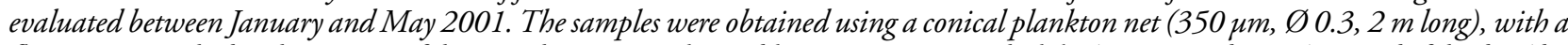
flow meter attached to the opening of the net. The categorical variables were: position in the lake (margin and center), period of the day (day and night) and depth (surface and bottom). The analysis was made with Fatorial Correspondence Analyses (FCA) using the larval density $\left(\right.$ (larvae. $\left.m^{-3}\right)$ as answer variable and the months as descriptors. The feeding activity of larvae was also studied. Most of them were protolarvae feeding in the margin of the lake, on the surface as well as on the bottom. These results indicated that Catalão Lake is an important nursery ground for to the larvae of fishes that spawn in the Solimöes River.

\section{KEY WORDS}

Solimões River; Catalão lake; fish-larvae; abundance; distribution; feeding.

${ }^{1}$ Instituto Nacional de Pesquisas da Amazônia - INPA

${ }^{2}$ Universidade Federal do Amazonas - UFAM 


\section{INTRODUÇÃO}

O período de enchente dos rios amazônicos é fundamental para a atividade reprodutiva de muitas espécies de peixes, principalmente dos Characiformes e Siluriformes (Araújo-Lima, 1984). Nesta fase do ciclo hidrológico, muitas espécies deslocamse dos tributários até a calha dos rios principais para desovarem. Um modelo bem conhecido de reprodução dos peixes migradores é proposto por Goulding (1980), no qual os peixes saem anualmente dos lagos, igarapés e rios de águas pobres para desovarem em ambientes considerados ricos em nutrientes. Por outro lado, também ocorrem desovas nos rios de águas consideradas pobres, como é o caso do rio Negro (Oliveira, 2003).

Embora os rios sejam favoráveis ao desenvolvimento de larvas de peixes, principalmente o rio Amazonas que é muito produtivo, no seu canal principal não há produção biológica suficiente para o sustento das larvas (Fisher, 1978; Forsberg, 1985). Por essa razão, é raro encontrar larvas de Characiformes em atividade alimentar na calha do rio Amazonas (Leite, 2000).

Enquanto as larvas possuem o saco vitelínico, elas são conduzidas pelas águas dos rios no seu canal principal até atingirem as áreas de inundação onde adquirem seu primeiro alimento exógeno (Leite \& Araújo-Lima, 2001). Nesses locais, fatores como a turbulência e a turbidez contribuem muito para que as larvas não tenham sucesso na captura de suas presas, principalmente porque elas não são boas nadadoras.

A maior concentração de larvas com alimento no tubo digestivo ocorre nas áreas de inundação recente, onde existem alimentos e formam-se abrigos naturais (Junk, 1973; Leite, 2000; Leite \& Araújo-Lima, 2002). Muitas dessas áreas estão na região de desembocadura dos canais que ligam os lagos aos rios e nas margens destes, nos primeiros meses de inundação.

O Lago Catalão situa-se próximo ao encontro das águas dos rios Solimões e Negro, área onde ocorrem desovas de muitas espécies de Characiformes (Araújo-Lima, 1984). O objetivo principal deste trabalho foi verificar a importância dessa proximidade para a dispersão e alimentação de larvas de peixes no Lago.

\section{MATERIAL E MÉTODOS}

\section{ÁREA DE ESTUDO}

O Lago Catalão situa-se próximo à confluência dos rios Solimões e Negro (Fig. 1), a 3o 09' 47"S e 059o 54' 29" W, com distância aproximada de $3.000 \mathrm{~m}$ do porto da CEASA, em Manaus. Como a maioria dos lagos de várzea amazônicos, o Lago Catalão aumenta ou diminui de tamanho de acordo com o nível dos rios adjacentes. O Lago Catalão normalmente conecta-se ao rio Solimões através de um curto carnal, após os primeiros meses de subida das águas. Por outro lago, raramente fica totalmente desconectado do rio Negro. Nas secas mais intensas, este lago resume-se a uma pequena área denominada "poção" e, mesmo assim, fica vertendo água para o rio Negro.

\section{AMOSTRAGEM}

Cada amostra consistiu de arrastes horizontais na superfície e fundo do lago com duração média de 3 minutos cada, utilizando rede de ictioplâncton cônico-cilíndrica com $350 \mu \mathrm{m}$ de malha, $\varnothing 0,3 \mathrm{~m}$ e $2 \mathrm{~m}$ de comprimento, armada na lateral da canoa a uma velocidade de $\pm 1 \mathrm{~m} \cdot \mathrm{s}^{-1}$. O volume de água filtrado foi calculado, usando um fluxômetro mecânico da marca General Oceanics, acoplado na boca da rede. As amostras foram tomadas mensalmente, no período de janeiro a maio de 2001, na margem e no centro do lago, tanto de dia quanto à noite, com duas repetiçōes cada, totalizando 90 amostras.

\section{PROCESSAMENTO DAS AMOSTRAS}

As amostras foram fixadas em formalina a $10 \%$ e depois transferidas para álcool a 70\%. A identificação das larvas foi baseada nos trabalhos de Araújo-Lima (1984; 1985), AraújoLima \& Donald (1988) e Nakatani et al. (2001) e os estágios do desenvolvimento larval foram baseados na nomenclatura proposta por Snyder (1976). As larvas foram classificadas por espécie e/ou grupo, estágio de desenvolvimento e separadas por turno (diurno ou noturno), profundidade (superfície ou fundo) e posição no lago (margem ou centro). Após esta avaliação, os dados foram agrupados para análise.

\section{ANÁLISE DOS DADOS}

A abundância das larvas foi obtida considerando a quantidade de larvas capturadas pelo volume de água filtrado. Os dados da estimativa foram transformados em larvas. $50 \mathrm{~m}^{-3}$.

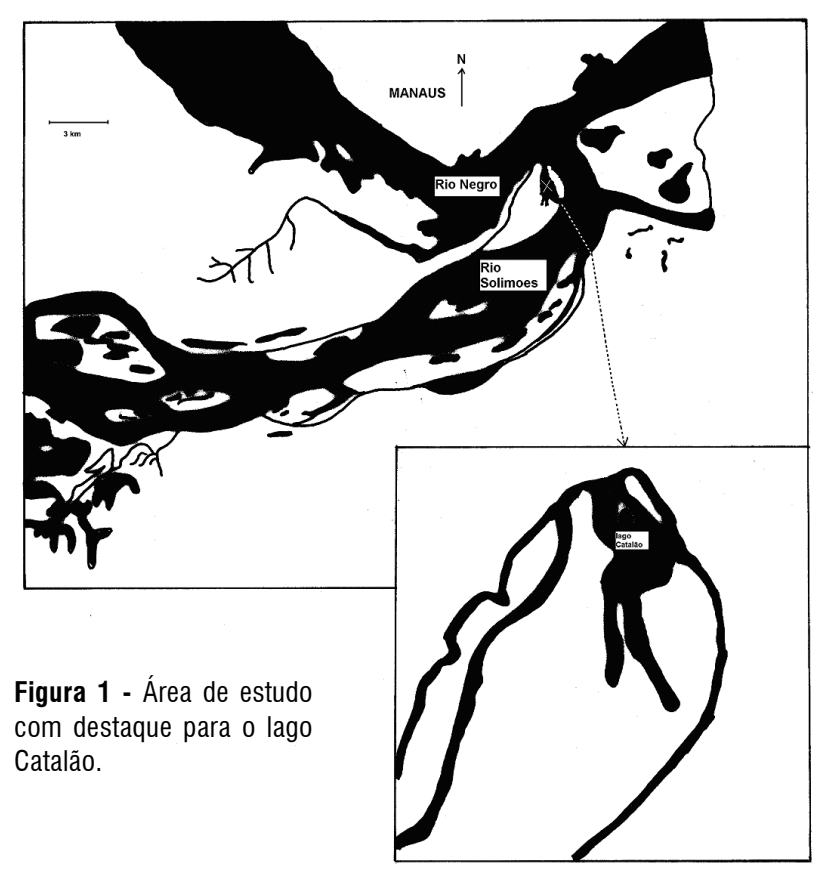


Para melhor compreensão, as unidades experimentais compostas por local, profundidade e turno foram codificadas da seguinte maneira: $\mathrm{MSD}=$ margem superfície dia; $\mathrm{MSN}=$ margem superfície noite; $\mathrm{MFD}=$ margem fundo dia; $\mathrm{MFN}=$ margem fundo noite; $\mathrm{CSD}=$ centro superfície dia; $\mathrm{CSN}=$ centro superfície noite; $\mathrm{CFD}=$ centro fundo dia e $\mathrm{CFN}=$ centro fundo noite.

Foi realizada uma Análise Fatorial de Correspondência (Ter Braak, 1995) para identificar os padrōes de ocorrência das larvas combinando os fatores: local do lago (margem - M e centro - C), profundidade (superfície - S e fundo - F) e turno (diurno - D e noturno - $\mathrm{N}$, em função de unidades experimentais, usando a densidade larval (larvas. $\mathrm{m}^{-3}$ ) como variável resposta e os meses de amostragem como variável descritora. Este tipo de análise é eficiente como técnica explanatória, e apresenta a vantagem de não exigir multinormalidade do conjunto de dados.

Para verificar se as larvas estavam em atividade alimentar, foi considerada apenas a presença ou a ausência de alimentos no tubo digestivo.

\section{RESULTADOS}

A primeira dimensão da Análise Fatorial de Correspondência - AFC (Fig. 2), discriminou a ocorrência das larvas entre o mês de janeiro e os demais, por unidade experimental, com um percentual de inércia de $58,15 \%$. Em janeiro, as larvas ocorreram com maior freqüência durante o dia, na superfície e na margem. A segunda dimensão da AFC explicou 32,70\% do arranjo e separou o mês de março, quando as larvas foram encontradas mais à noite e na superfície, tanto na margem como no centro do lago. As larvas apresentaram maior concentração na área central do lago nos meses de fevereiro, abril e maio.

A composição taxonômica das larvas capturadas e a abundância média mensal estão representadas na Tabela 1 . Dentre os Characiformes foram identificadas larvas de Anodus

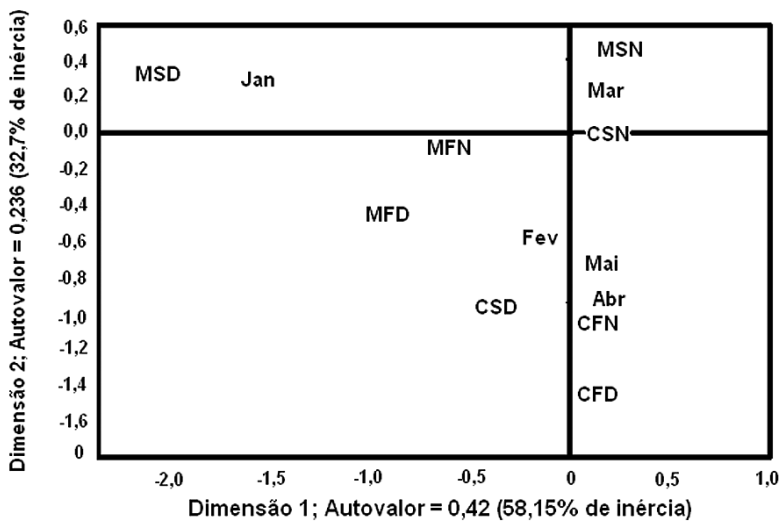

Figura 2 - Gráfico da Análise de Correspondência entre os meses e os locais de captura das larvas de peixes no lago Catalão. $\mathrm{M}=$ margem, $\mathrm{C}=$ centro, $S=$ superfície, $F=$ fundo, $D=$ dia e $N=$ noite. elongatus (Hemiodontidae) Mylossoma aureum e Mylossoma duriventre (Serrasalmidae), Prochilodus nigricans e Semaprochilodus insignis (Prochilodontidae), Rhythiodus microlepis e Schizodon fasciatum (Anostomidae), Triportheus elongatus, Triportheusflavus e Triportheus sp. (Characidae). Outras larvas de Characiformes que não foram identificadas em nível de gênero ou espécie pertencem às famílias: Curimatidae, Hemiodontidae e Cynodontidae. Das demais ordens ocorreram Colomesus asellus (Tetraodontiformes); Sorubim lima e Auchenipterus nuchalis (Siluriformes); e Plagioscion spp (Perciformes). Foram identificadas, ainda, espécies pertencentes à ordem Clupeiforme.

Ocorreram oscilaçōes consideráveis na abundância das larvas ao longo dos meses estudados em níveis intra e interespecíficos (Tabela 1). No mês de janeiro, as larvas de $A$. elongatus foram as que apresentaram maior abundância, com mais de 340 larvas. $50 \mathrm{~m}^{-3}$, enquanto que as demais espécies corresponderam a menos de 50 larvas. $50 \mathrm{~m}^{3}$.

Em fevereiro, larvas de curimatídeos, juntamente com larvas de T. flavus e Triportheus sp., foram as de maior abundância, com mais de 100 larvas. $50 \mathrm{~m}^{-3}$. Em março, os curimatídeos atingiram densidade superior a 1000 larvas. $50 \mathrm{~m}^{-3}$, seguidos por $M$. duriventre e $M$. aureum. (Tabela 1). No mês de abril, a densidade das larvas de curimatídeos decresceu para 60 larvas. $50 \mathrm{~m}^{-3}$, enquanto que as larvas das demais espécies mantiveram-se sempre abaixo de 50 larvas. $50 \mathrm{~m}^{-3}$. Em maio, a densidade das larvas foi inferior a 40 larvas. $50 \mathrm{~m}^{-3}$, na maioria das espécies (Tabela 1).

Tabela 1 - Abundância mensal das larvas de peixes. $50 \mathrm{~m}^{-3}$ no Lago Catalão entre Janeiro e Maio de 2001, correspondente ao período de enchente dos rios Solimões/Amazonas e Negro.

\begin{tabular}{|cccccc}
\hline Espécies & Janeiro & Fevereiro & Março & Abril & Maio \\
\hline Larvas de Engraulididae & 16,4 & 7,1 & 5 & 2,7 & 0 \\
\hline Larvas de Clupeidae & 15,2 & 6,5 & 4,2 & 2,7 & 0 \\
\hline Triportheus elongates & 4,4 & 13,0 & 17,5 & 2,7 & 0 \\
\hline Triportheus flavus & 13 & 114,4 & 34,5 & 5,9 & 40,8 \\
\hline Triportheus sp. & 0 & 103,2 & 0 & 0 & 0 \\
\hline Anodus elongatus & 353,6 & 20,3 & 5 & 0 & 0 \\
\hline Schizodon fasciatum & 0 & 40,5 & 11,7 & 9,2 & 5,7 \\
\hline Rhytiodus microlepis & 0 & 24,5 & 0 & 0 & 0 \\
\hline Prochilodus nigricans & 0 & 22,6 & 58 & 5,43 & 0 \\
\hline Semaprochilodus insignis & 8,7 & 26,4 & 317,5 & 5,43 & 0 \\
\hline Larvas de Curimatidae & 46,4 & 106,9 & 1063,1 & 65,2 & 7,9 \\
\hline Larvas de Hemiodontidae & 13 & 3,2 & 9,1 & 7,61 & 0 \\
\hline Mylossoma aureum & 6,5 & 30,4 & 146 & 0 & 0 \\
\hline Mylossoma duriventre & 4,4 & 55,0 & 210 & 35,1 & 11,6 \\
\hline Larvas de Cynodontidae & 4,4 & 10,3 & 6,1 & 5,1 & 0 \\
\hline Sorubim lima & 0 & 0 & 3,3 & 6,9 & 3,4 \\
\hline Auchenipterus nuchalis & 7,3 & 8,0 & 13 & 39,4 & 23,3 \\
\hline Plagioscion spp. & 34,8 & 3,2 & 3,3 & 2,7 & 3,4 \\
\hline Colomesus asellus & 0 & 3,9 & 7,3 & 9,2 & 3,0 \\
\hline
\end{tabular}


Tabela 2 - Larvas capturadas no Lago Catalão de Janeiro a Maio de 2001. As letras representam posições no Lago ou período do dia em que foram capturadas: M - margem do lago, C - centro do lago, S - superfície, F - fundo, D - dia, N - noite. Os estágios larvais são indicados por PT(protolarva), MS (mesolarva) e MT (metalarva). Os números significam porcentagem de larvas com alimento: 0 - todos sem alimento, 1 (1 a 25\%), 2 (25,1 a 50\%), 3 (50,1 a 75\%) e 4 (acima de 75\%) com alimento respectivamente. Traços indicam a não captura de larvas nos respectivos estágios.

\begin{tabular}{|c|c|c|c|c|c|c|c|c|c|c|c|c|c|c|c|c|c|c|c|c|c|c|c|}
\hline \multirow{2}{*}{$\begin{array}{c}\text { Posição } \\
\text { Espécie/Estágio }\end{array}$} & \multicolumn{3}{|c|}{ MSD } & \multicolumn{3}{|c|}{ MSN } & \multicolumn{3}{|c|}{ MFD } & \multicolumn{3}{|c|}{ MFN } & \multicolumn{2}{|c|}{ CSD } & \multicolumn{3}{|c|}{ CSN } & \multicolumn{3}{|c|}{ CFD } & \multicolumn{3}{|c|}{ CFN } \\
\hline & PT & MS & MT & PT & MS & MT & PT & MS & MT & PT & MS & MT & PT & MT & PT & MS & MT & PT & MS & MT & PT & MS & MT \\
\hline Larvas de Engraulidídae & 1 & - & 0 & 1 & 1 & 1 & 2 & 0 & 0 & 0 & 2 & 0 & 1 & 4 & - & 0 & - & 2 & - & - & 0 & 2 & 0 \\
\hline Larvas de Clupeídae & 0 & 4 & - & 1 & 2 & 0 & 0 & 0 & - & - & 2 & - & - & - & - & - & - & - & - & - & - & - & - \\
\hline Triportheus elongates & 0 & - & - & 2 & 4 & 4 & 1 & 3 & 4 & 1 & 3 & 3 & - & - & 0 & 2 & 3 & 0 & 2 & 3 & 1 & 2 & 2 \\
\hline Triportheus flavus & - & - & - & 3 & - & - & - & - & - & 3 & - & - & - & - & 3 & 4 & - & 0 & - & - & - & - & - \\
\hline Anodus elongatus & 2 & 4 & - & 1 & 2 & - & 2 & 2 & - & 2 & 1 & 0 & 2 & 0 & 1 & 0 & 0 & 4 & 0 & 0 & 0 & 0 & 0 \\
\hline Schizodon fasciatum & - & - & - & - & - & - & - & - & - & - & - & - & - & - & - & - & - & - & 2 & - & - & - & - \\
\hline Rhytiodus microlepis & - & - & - & 2 & 0 & - & 3 & 4 & 4 & 4 & - & - & - & - & - & - & - & - & - & - & - & - & - \\
\hline Prochilodus nigricans & - & - & - & 1 & - & - & - & - & - & 2 & - & - & - & - & - & 2 & - & - & - & - & 0 & - & - \\
\hline Semaprochilodus insignis & - & - & - & - & 0 & - & - & - & - & 4 & 4 & 4 & - & - & - & 2 & - & - & 4 & - & - & 0 & - \\
\hline Larvas de Hemiodontidae & - & - & - & 1 & - & - & - & - & - & 2 & - & - & 0 & - & 0 & - & - & 0 & - & - & 0 & - & - \\
\hline Mylossoma aureum & 3 & - & 4 & 2 & 4 & & 4 & - & - & 2 & 4 & - & - & - & 1 & 4 & - & 1 & - & - & 0 & - & - \\
\hline Mylossoma duriventre & 2 & - & - & 2 & 3 & 4 & 3 & - & - & 2 & 2 & - & 4 & - & 1 & - & - & 1 & - & - & 1 & - & - \\
\hline Larvas de Cynodontidae & - & - & - & 0 & 3 & - & - & - & - & - & 3 & - & - & - & 2 & 2 & - & 4 & 2 & - & 3 & 0 & - \\
\hline Sorubim lima & 0 & - & - & 1 & 4 & 4 & 1 & - & - & 2 & 3 & 2 & - & - & 1 & 1 & 4 & 0 & 2 & - & 0 & - & - \\
\hline Auchenipterus nuchalis & - & - & - & 1 & - & - & 2 & - & - & 2 & - & - & - & - & 1 & - & - & 1 & - & - & 0 & - & - \\
\hline Plagioscion spp. & 2 & - & - & 2 & - & - & 2 & - & - & 4 & 3 & - & 2 & - & 4 & - & 4 & - & 3 & 4 & 0 & - & 4 \\
\hline Colomesus asellus & 3 & 0 & - & 4 & 0 & - & 3 & 4 & - & 0 & 0 & 0 & 2 & 0 & 2 & 0 & 0 & 0 & 0 & 0 & 0 & 0 & 0 \\
\hline
\end{tabular}

Ao longo de todo o período estudado, a maioria das larvas capturadas encontrava-se no estágio de protolarva e a maior freqüência de larvas com alimento no trato digestivo ocorreu na margem do lago, tanto na superfície quanto no fundo. Menor freqüência de larvas ocorreu no centro do lago à noite, tanto na superfície quanto no fundo (Tabela 2).

Durante o dia, as larvas capturadas no centro do lago alimentaram-se com mais freqüência na superfície, quando comparadas com as larvas capturadas no fundo (Teste $\mathrm{t}, \mathrm{gl}=8$, $\mathrm{t}$ $=2,895, \mathrm{p}=0,020)$. À noite, houve maior proporção de larvas alimentando-se na margem do lago, quando comparadas com aquelas do centro do lago (Teste $\mathrm{t}, \mathrm{gl}=8, \mathrm{t}=2,510, \mathrm{p}=0,036$ ).

\section{DISCUSSÃO}

\section{ABUNDÂNCIA}

A maioria das larvas encontradas no Lago Catalão pertencia à ordem Characiformes. Essa prevalência já havia sido observada por Araújo-Lima \& Oliveira (1998), no rio Solimões/Amazonas, onde atingiram $39,5 \%$ da abundância total. No rio Negro, ao contrário, essa ordem contribuiu com apenas $5 \%$ da abundância total, de acordo com Oliveira (2003). A comparação destes dois resultados confere ao Lago Cataláo um perfil mais relacionado ao rio Solimōes/Amazonas.
Os Characiformes encontrados no Lago Catalão, em sua maioria, pertencem a espécies que se encaixam nos padrões de migração para desova sugerida por Goulding (1980) para o rio Madeira ou, também, no padrão de migração do gênero Semaprochilodus spp. no rio Solimōes/Amazonas e no rio Negro descrito por Ribeiro (1983). Em ambos os casos, os autores mostraram a tendência dos peixes adultos migrarem dos rios de águas pobres para os rios de águas ricas em nutrientes para efetuarem a desova.

Os vestígios dessa migração em direção rio Amazonas também foram observados por Lima \& Araújo-Lima (2004), quando encontraram adultos de $M$. aureum, $M$. duriventre, $C$. macropomum, A. elongatus, T. elongatus, B. amazonicus, S. insignis, $S$. taeniurus e $P$. nigricans em nove rios com diferentes tipos de água, porém as larvas e juvenis dessas espécies foram encontrados somente nos rios de água branca.

Reportando-se a uma área muito próxima ao Lago Catalão, Araújo-Lima (1984) mostrou que a densidade de larvas na região do encontro das águas foi sempre menor na parte em que havia maior influência do rio Negro, reforçando, dessa forma, os modelos e dados descritos anteriormente. $\mathrm{O}$ mesmo autor também observou maior densidade de larvas de Characiformes na margem do rio na costa do Catalão, um pouco acima do 
encontro das águas no rio Solimões, constatando que as desovas estavam ocorrendo naquela área.

Dois fatores principais podem agir, em conjunto, para que as larvas atinjam as áreas inundadas e o Lago Catalão provenientes do canal principal do rio ou de suas margens: a direção das correntes e o pulso de inundação (Araújo-Lima, 1984; Junk et al. 1989). Com o aumento do volume de água, é restabelecido um canal que permite a passagem direta do rio Solimōes para o Lago Catalão, diferente do que ocorre no período de água baixa, quando há um isolamento entre o rio e o referido lago.

Além do canal, outras vias de acesso ao lago se abrem através da vegetação inundada, sendo encontrada uma grande quantidade de juvenis, principalmente, de sardinhas (Triportheus spp.) e de jaraquis (Semaprochilodus spp.) (Leite, Obs. Pessoal).

Araújo-Lima (1984) afirmou que a densidade das larvas no rio atingiu o pico máximo quando a velocidade de subida do nível da água atingia o máximo valor. No lago Catalão, foi observado que entre 20 e $22 \mathrm{~m}$ acima do nível do mar, correspondente a janeiro e fevereiro de 2001, houve um incremento no número de espécies no local e muitas delas ocorreram em altas densidades.

Além do evento da entrada de larvas provenientes do rio Solimões para o Lago Catalão, muitas espécies reproduziram no próprio lago, o que também contribuiu para a ocorrência de picos isolados de larvas de espécies não migradoras.

DISTRIBUIÇÃO DAS LARVAS EM FUNÇÃO DE SUA ATIVIDADE ALIMENTAR

As larvas encontradas no Lago Catalão estavam em atividade alimentar em todos os estágios de desenvolvimento. Este comportamento difere dos resultados obtidos por Oliveira (1996), Leite (2000) e Leite \& Araújo-Lima (2002), que observaram pouca ou nenhuma atividade alimentar das larvas no canal do rio. Uma exceção foi observada em larvas de $B$. amazonicus, que se aproveitam do seu maior tamanho, para se alimentarem de larvas de outras espécies de Characiformes na calha do rio, em locais distante das margens.

De acordo com Moura (1998), as larvas que se alimentaram no canal do rio pertenciam, principalmente, às famílias Pimelodidae e Clupeidae, e na maioria delas, correspondiam ao estágio metalarva. Estas larvas estavam comendo, quase que exclusivamente, larvas de Characiformes, com apenas um caso de ingestão de larvas de Tetraodontiformes e um caso de Siluriformes.

No presente trabalho, as mesolarvas e metalarvas apresentaram maior proporção de indivíduos se alimentando do que as protolarvas, que ocorreram em maior número. A menor participação das larvas coletadas nos estágios meso e metalarva pode ser um reflexo da sua capacidade de escape das redes, ou então, como conseqüência de estarem explorando áreas de macrófitas flutuantes ou não, onde não foi possível passar a rede de ictioplâncton.
A maior atividade alimentar das larvas no Lago Catalão foi observada nas margens, quando comparada às estaçōes do centro do lago. Este comportamento de maior atividade alimentar nas margens, ou em áreas mais próximas a um determinado substrato, corrobora o que foi descrito por Leite (2000).

Algumas espécies que comiam na superfície à noite, não tinham alimento no estômago durante o dia e vice-versa. De acordo com Zaret (1984), este comportamento pode ser entendido como uma estratégia das larvas no processo de interaçôes que existem entre os peixes e o zooplâncton.

Em conclusão, o Lago Catalão é uma área importante para o desenvolvimento de larvas de peixes, principalmente das espécies que desovam no rio Solimões, acima do encontro das águas com o rio Negro. Este lago também serve como área de dispersão e alimentação dos peixes nesta fase do desenvolvimento. Além disso, muitas espécies residentes reproduzem-se no próprio lago durante a enchente dos rios.

\section{BIBLIOGRAFIA CITADA}

Araújo-Lima, C.A.R.M. 1984. Distribuição espacial e temporal de larvas de Characiformes em um setor do rio Solimóes/Amazonas próximo a Manaus, $A M$. Dissertação de Mestrado, Instituto Nacional de Pesquisas da Amazônia/Fundação Universidade do Amazonas, Manaus, Amazonas. 84pp.

Araújo-Lima, C.A.R.M. 1985. Aspectos biológicos de peixes amazônicos. V. Desenvolvimento larval do jaraqui-escama grossa, Semaprochilodus insignis (Characiformes, Pisces) da Amazônia central. Rev. Brasil. Biol., 45(4): 423-443.

Araújo-Lima, C.A.R.M.; Donald, E. 1988. Número de vértebras de Characiformes do rio Amazonas e seu uso na identificação de larvas do grupo. Acta Amazonica, 18: 351-358.

Araújo-Lima, C.A.R.M.; Oliveira, E.C. 1998. Transport of larval fish in the Amazon. J. Fish Biol., 53(supl. A): 297-306.

Araújo-Lima, C.A.R.M; Silva, J.V.V; Petry, P; Oliveira, E.C; Moura, S.M.L. 2001. Diel variation of larval fish abundance in the Amazon and Rio Negro. Brazil. J. Biol., 61(3): 357-362.

Fisher, T.R. 1978. Plâncton e produção primária em sistemas aquáticos da bacia Amazônica Central. Acta Amazonica, 8(supl.): 43-54.

Forsberg, B.R. 1985. The fate of planktonic primary production. Limnology and Oceanography, 30(4):807-819.

Goulding, M. 1980. The fishes and the forest. University of California Press, Los Angeles, USA. 200pp.

Junk, W.J. 1973. Investigations on the ecology and productionbiology of the "floating-meadows" (Paspalo chinochloetum) on the Midle Amazon. Part II - The aquatic fauna in the root-zone of floating vegetation. Amazoniana, 4(1): 9-12.

Junk, W.J.; Bayley, P.B.; Sparks, R.E. 1989. The flood pulse concept in river-floodplain systems, p. 110-127. In: D. P. Dodge (Ed.) Proceedings of the International Large River symposium. Can. Spec. Publi. Fish. Aquat. Sci., 106. 
Leite, R.G. 2000. Alimentação e fontes autotróficas de energia utilizadas pelas larvas de peixes do rio Solimóes/Amazonas e suas áreas inundáveis. Tese de Doutorado, Instituto Nacional de Pesquisas da Amazônia/Universidade Federal do Amazonas, Manaus, Amazonas, AM. 118pp.

Leite, R.G.; Araújo-Lima, C.A.R.M. 2002. Feeding of the Brycon cephalus, Triportheus elongatus and Semaprochilodus insignis (Osteichthyes, Characiformes) larvae in Solimôes/Amazonas River and floodplain areas. Acta Amazonica, 32(3): 129-147.

Lima, A.C; Araújo-Lima, C.A.R.M. 2004. The distribution of larval and juvenile fishes in Amazonian rivers of different nutrient status. Freshwater Biology, 49: 1-14.

Moura, S.M.L. 1998. Predação de larvas de peixes no rio Amazonas. Dissertação de Mestrado, Instituto Nacional de Pesquisas da Amazônia/Universidade Federal do Amazonas, Manaus, Amazonas. 28pp.

Nakatani, K.; Agostinho, A.A.; Baumgartner, G.; Bialetzki, A.; Sanches, P.V.; Makrakis, M.C.; Pavanelli, C.S. 2001. Ovos e larvas de peixes de água-doce: desenvolvimento e manual de identificação. EDUEM, Maringá, Paraná. 378pp.

Oliveira, E.C de. 1996. Distribuição das Larvas de Mylossoma aureum e M. duriventre (Pisces: Serrasalmidae) no rio Amazonas. Dissertação de Mestrado, Instituto Nacional de Pesquisas da Amazônia, Manaus, AM. 28pp.
Oliveira, E.C. 2003. Distribuiçāo e abundância do ictioplâncton na área da Estação Ecológica de Anavilhanas, Rio Negro, Amazonas, Brasil. Tese de Doutorado, Instituto Nacional de Pesquisas da Amazônia, Manaus, Amazonas. 157pp.

Ribeiro, M.C.L.B. 1983. As migraçōes dos jaraquis (Pisces, Prochilodontidae) no Rio Negro, Amazonas, Brasil. Dissertação de Mestrado, Instituto Nacional de Pesquisas da Amazônia, Manaus, Amazonas. 192pp.

Snyder, D.E. 1976. Terminologies for intervals of larval fish development.In: Boreman, J., (Ed.). Great Lakes fish egg and larvae identification. U.S. Fish Wild. Ser.: p. 41-58.

Ter Braak, C.J.F. 1995. Data analysis in community and landscape ecology, In: Jongman, R. H.G.; Braak Ter, C.J.F; Tongeren, O.F.R. Van (Eds.), Ordination, Cambridge University Press, Cambridge. p. 91-173.

Zaret, T.M. 1984. Fish/zooplankton interactions in amazonian floodplain lakes. Verh. Internat. Verein. Limnol., 22: 1305-1309.

Recebido em 14/10/2003

Aceito em 11/09/2006 